\title{
Education and Professional Standards Committee (EPSC) Program 2013 - 2015
}

\section{"Education is the most powerful weapon which you can use to change the world" (Nelson Mandela)}

Technological progress opened new perspectives to ultrasound, new clinical applications are emerging therefore education and subsequently preparation and updating of the US knowledge is crucial. The EPSC has the aim to plan, initiate and monitor international co-operative studies on training in medical ultrasound; to propose and formulate scientific sessions on all aspects of ultrasound training and accreditation for inclusion in the EUROSON Congresses, Euroson Schools and Endorsed Courses; to arrange meetings, conference sessions and training courses as necessary to promote ultrasound education. The aim is to continue with the tasks already started such as:

1. The Integration of European sonographic courses; templates, rules and uniformity of quality of teaching has been revised and changed where necessary.

2. The improvement of e-learning. EFSUMB has an online educational portal with cases and video presentations and an ATLAS of images to accompany the Elastography, CEUS Liver and non Liver Guidelines. It also offers a post-graduate web course and links to useful web pages.

3. To complete the phase of preparation of the common curriculum of European Ultrasound Learning Centres starting with the establishment of the first centres for initiating a promotional and experimental phase in order to disseminate them and to make them attractive to users.

4. To continue to better define and promote teaching of ultrasound at the university co-operating with the universities which have already implemented this.

Vito Cantisani, EPSC Chairman and Committee - Dieter Nuernberg, Radu Badea, Eugenio Cerezo, Hilde Berner Hammer, Adnan Kabaalioglu, Helmut Prosch and Andreas Serra. 